\title{
Bounded point derivations and functions of bounded mean oscillation
}

\author{
Stephen Deterding * \\ West Liberty University, \\ West Liberty, WV, USA
}

\begin{abstract}
Let $X$ be a subset of the complex plane and let $A_{0}(X)$ denote the space of VMO functions that are analytic on $X . A_{0}(X)$ is said to admit a bounded point derivation of order $t$ at a point $x_{0} \in \partial X$ if there exists a constant $C$ such that $\left|f^{(t)}\left(x_{0}\right)\right| \leq C|| f \|_{B M O}$ for all functions in $V M O(X)$ that are analytic on $X \cup\left\{x_{0}\right\}$. In this paper, we give necessary and sufficient conditions in terms of lower 1-dimensional Hausdorff content for $A_{0}(X)$ to admit a bounded point derivation at $x_{0}$. These conditions are similar to conditions for the existence of bounded point derivations on other functions spaces.
\end{abstract}

\section{Introduction}

Let $X$ be a subset of the complex plane and suppose that $f$ is analytic on a neighborhood of $X$. If $x_{0}$ is an interior point of $X$, then it follows from the Cauchy estimates that $f^{\prime}\left(x_{0}\right)$ cannot be too large relative to the size of $f$. To be precise, in this situation there exists a positive number $C$ such that for every function $f$ analytic on $X,\left|f^{\prime}\left(x_{0}\right)\right| \leq C|| f \|_{\infty}$, where $\|f\|_{\infty}$ is the supremum norm of $f$ on $X$. However, this is not true if $x_{0}$ is a boundary point. For example, if $X$ is the unit disk then the sequence $f_{n}=z^{n}$ has supremum norm 1 on $X$ for all $n$ but $f_{n}^{\prime}(1) \rightarrow \infty$ as $n \rightarrow \infty$.

${ }^{*}$ email: Stephen.Deterding@westliberty.edu 
Runge's theorem states that every analytic function on a neighborhood of $X$ can be approximated uniformly by rational functions with poles off $X$, so we can suppose that the function $f$ is a rational function with poles off $X$. Let $R(X)$ denote the closure of the rational functions with poles off $X$ in the uniform norm. For non-negative integer values of $t$ we say that $R(X)$ admits a bounded point derivation of order $t$ at $x_{0}$ if there exists a constant $C$ such that for all rational functions $f$ with poles off $X,\left|f^{(t)}\left(x_{0}\right)\right| \leq C\|f\|_{\infty}$. From the above discussion, we see that $R(X)$ admits a bounded point derivation at all interior points of $X$, while at a boundary point $x_{0}$ the derivatives of analytic functions are bounded in the uniform norm if and only if $R(X)$ admits a bounded point derivation at $x_{0}$.

One can define bounded point derivations for the closure of rational functions in other Banach spaces as well. If $B$ is a Banach space with norm $\|\cdot\|_{B}$ and $R_{B}(X)$ denotes the closure of rational functions in $B$ then we say that $R_{B}(X)$ admits a bounded point derivation of order $t$ at $x_{0}$ if there exists a constant $C$ such that for all rational functions $f$ with poles off $X$, $\left|f^{(t)}\left(x_{0}\right)\right| \leq C\|f\|_{B}$

Necessary and sufficient conditions for the existence of bounded point derivations have been determined for the closure of rational functions in the following Banach spaces $B$ : $C(X)$, $L^{P}(X), \operatorname{Lip} \alpha(X)$, and in this paper we will determine these conditions for $V M O(X)$, the space of functions of vanishing mean oscillation. Our main theorem is the following.

Theorem 1. Let $X$ be a subset of the complex plane and let $A_{0}(X)$ denote the space of VMO functions that are analytic on $X$. Choose $x_{0} \in \partial X$ and let $A_{n}=\left\{2^{-(n+1)} \leq\left|z-x_{0}\right| \leq 2^{-n}\right\}$. Then there exists a bounded point derivation of order $t$ on $A_{0}(X)$ at $x_{0}$ if and only if

$$
\sum_{n=1}^{\infty} 2^{(t+1) n} M_{*}^{1}\left(A_{n} \backslash X\right)<\infty
$$

where $M_{*}^{1}$ denotes lower 1-dimensional Hausdorff content.

In the next section, we review some of the results of bounded point derivations on other function spaces to show how the conditions for bounded point derivations on $A_{0}(X)$ compare with those for the other Banach spaces. In section 3 we review the concepts of BMO spaces and Hausdorff contents, and in section 4 we prove Theorem 1. In the last section, Theorem 1 is used to provide examples of sets with and without bounded point derivations on $A_{0}(X)$. 


\section{Bounded Point Derivations on Other Function Spaces}

Let $U$ be an open subset of the complex plane and let $0<\alpha<1$. A function $f: U \rightarrow \mathbb{C}$ satisfies a Lipschitz condition with exponent $\alpha$ on $U$ if there exists $k>0$ such that for all $z, w \in U$

$$
|f(z)-f(w)| \leq k|z-w|^{\alpha} .
$$

Let $\operatorname{Lip} \alpha(U)$ denote the space of functions that satisfy a Lipschitz condition with exponent $\alpha$ on $U$. $\operatorname{Lip} \alpha(U)$ is a Banach space with norm given by $\|f\|_{L i p \alpha(U)}=\sup _{U}|f|+k(f)$, where $k(f)$ is the smallest constant that satisfies (1).

An important subspace of $\operatorname{Lip} \alpha(U)$ is the little Lipschitz class, lip $\alpha(U)$, which consists of those functions in $\operatorname{Lip} \alpha(U)$ such that

$$
\lim _{\delta \rightarrow 0^{+}} \sup _{0<|z-w|<\delta} \frac{|f(z)-f(w)|}{|z-w|^{\alpha}}=0
$$

Let $A_{\alpha}(U)$ denote the space of lip $\alpha$ functions that are analytic on $U . A_{\alpha}(U)$ is said to admit a bounded point derivation of order $t$ at $x_{0} \in \partial X$ if there exists a constant $C$ such that

$$
\left|f^{(t)}\left(x_{0}\right)\right| \leq C|| f \|_{\text {Lip }}
$$

whenever $f \in \operatorname{lip} \alpha(\mathbb{C})$ is analytic in a neighborhood of $U \cup\left\{x_{0}\right\}$. In [10] Lord and O'Farrell determined necessary and sufficient conditions for $A_{\alpha}(U)$ to admit a bounded point derivation at $x_{0}$ in terms of lower dimensional Hausdorff content, which is defined in the next section. In that paper, what we call $A_{\alpha}(U)$ is denoted by $a_{\alpha}(U)$ since $A_{\alpha}(U)$ is used to denote the space of Lip $\alpha$ functions that are analytic on $U$. Their result is the following [10, Theorem 1.2].

Theorem 2. There exists a bounded point derivation of order $t$ on $A_{\alpha}(U)$ at $x_{0}$ if and only if

$$
\sum_{n=1}^{\infty} 2^{(t+1) n} M_{*}^{1+\alpha}\left(A_{n} \backslash U\right)<\infty
$$

where $M_{*}^{1+\alpha}$ denotes lower $(1+\alpha)$-dimensional Hausdorff content. 
Theorem 1 and Theorem 2 both involve series of Hausdorff contents of annuli. These are similar to existence theorems for bounded point derivations on other function spaces such as Hallstrom's theorem for $R(X)$ [5, Theorem 1, $\left.1^{\prime}\right]$, Hedberg's theorem for $R^{p}(X), p>2[7$, Theorem 2], and the theorem of Fernstrom and Polking for $R^{2}(X)[3$, Theorem 6]. In addition, O'Farrell has recently proven a similar theorem to Theorem 2] [12, Theorem 3.7] for functions belonging to negative Lipschitz classes in which $-1<\alpha<0$. Notably, this excludes the case of $\alpha=0$. As we will see the case of $\alpha=0$ can be identified with the space of analytic VMO functions.

\section{Bounded Mean Oscillation and Hausdorff Content}

Let $f \in L_{\text {loc }}^{1}(\mathbb{C})$ and let $Q$ be a cube in the complex plane with area $|Q|$. The mean value of $f$ on $Q$, denoted by $f_{Q}$, is

$$
f_{Q}=\frac{1}{|Q|} \int_{Q} f(z) d A
$$

and the mean oscillation of $f$ on a cube $Q$, denoted by $\Omega(f, Q)$, is

$$
\Omega(f, Q)=\frac{1}{|Q|} \int_{Q}\left|f(z)-f_{Q}\right| d A
$$

The function $f$ is said to be of bounded mean oscillation if

$$
\|f\|_{*}=\sup _{Q} \Omega(f, Q)<\infty
$$

where the supremum is taken over all cubes $Q$ in $\mathbb{C}$. Let $B M O(\mathbb{C})$ denote the set of functions of bounded mean oscillation and let $B M O(X)=\left\{\left.f\right|_{X}: f \in B M O(\mathbb{C})\right\}$. Let $\|f\|_{X}=$ inf $\|f\|_{*}$, where the infimum is taken over all functions $F$ such that $F=f$ on $X .\|f\|_{X}$ is a seminorm on $B M O(X)$, which vanishes only at the constant functions. If we let $\|f\|_{B M O(X)}=\|f\|_{X}+$ $\left|\int_{X} f(z) d A\right|$, then $\|f\|_{B M O(X)}$ defines a norm on $B M O(X)$.

An important subspace of $B M O(X)$ is $V M O(X)$, the space of functions of vanishing mean oscillation. For $f$ in $B M O(X)$ and $\delta>0$ let 


$$
\Omega_{f}(\delta)=\sup _{Q}\{\Omega(f, Q): \text { radius } \mathrm{Q} \leq \delta\}
$$

$V M O(X)$ consists of those functions in $B M O(X)$ which satisfy $\Omega_{f}(\delta) \rightarrow 0$ as $\delta \rightarrow 0^{+}$for all cubes $Q \in \mathbb{C}$. Let $A_{0}(X)$ denote the space of restrictions to $X$ of $V M O$ functions which are analytic on a neighborhood of $X$. Alternately, $f$ is a function of bounded mean oscillation on a set $X$ if and only if there exists a constant $K$ such that for every cube $Q \subseteq X$ and some constant $c_{Q}$, the inequality

$$
\int_{Q}\left|f(z)-c_{Q}\right| d A \leq K r^{2}
$$

holds where $r$ is the length of the edge of $Q$ [8]. This is similar to an alternate characterization of $\operatorname{Lip} \alpha$ functions. $f \in \operatorname{Lip} \alpha(X)$ if and only if there exists a constant $K$ such that for every cube $Q \subseteq X$ and some constant $c_{Q}$, the inequality

$$
\int_{Q}\left|f(z)-c_{Q}\right| d A \leq K r^{2+\alpha}
$$

holds where $r$ is the length of the edge of $Q\left[11\right.$. In this way $A_{0}(X)$ can be seen as the limit as $\alpha \rightarrow 0$ of $A_{\alpha}(X)$. Thus we say that $A_{0}(X)$ admits a bounded point derivation of order $t$ at $x_{0} \in \partial X$ if there exists a constant $C$ such that

$$
\left|f^{(k)}\left(x_{0}\right)\right| \leq C\|f\|_{B M O(X)}
$$

whenever $f \in V M O(\mathbb{C})$ is analytic in a neighborhood of $X \cup\left\{x_{0}\right\}$.

We saw in the last section that bounded point derivations on $A_{\alpha}(U)$ are characterized by lower $(1+\alpha)$ dimensional Hausdorff content, which suggests that bounded point derivations on $A_{0}(X)$ are characterized by lower 1-dimensional Hausdorff content. Some other examples of the connection between $V M O$ and lower 1-dimensional Hausdorff content in the context of rational approximation can be found in the paper of Verdera [13] (Here what we call $A_{0}(X)$ is denoted by $\left.V M O_{a}(X)\right)$, the paper of Boivin and Verdera [1], and the paper of Bonilla and Fariña [2]. 
We now define the lower 1-dimensional Hausdorff content. A measure function is an increasing function $h(t), t \geq 0$, such that $h(t) \rightarrow 0$ as $t \rightarrow 0$. If $h$ is a measure function then define

$$
M^{h}(E)=\inf \sum_{j} h\left(r_{j}\right)
$$

where the infimum is taken over all countable coverings of $E$ by squares with sides of length $r_{j}$. The lower 1-dimensional Hausdorff content of $E$, denoted $M_{*}^{1}(E)$ is defined by

$$
M_{*}^{1}(E)=\sup M^{h}(E)
$$

where the supremum is taken over all measure functions $h$ such that $h(t) \leq t$ and $h(t) t^{-1} \rightarrow 0$ as $t \rightarrow 0^{+}$. Furthermore, the infimum can be taken over countable coverings of $E$ by dyadic squares [4, pg. 61, Lemma 1.4]. It also follows from the definition that lower 1-dimensional Hausdorff content is subadditive; that is, $U \subseteq V$ implies $M_{*}^{1}(U) \leq M_{*}^{1}(V)$.

\section{The main result}

For the proof of Theorem 1, we will need the following lemma.

Lemma 3. Let $E_{j}, j=1, \ldots, n$ be sets and let $E=\bigcup_{j=1}^{n} E_{j}$. Let $\psi=\sum_{j=1}^{n} \psi_{j}$, where each $\psi_{j}$ has support on $E_{j}$. Then

$$
\int_{E} \psi d A=\sum_{j=1}^{n} \int_{E_{j}} \psi_{j} d A
$$

Proof. We first prove the case of $n=2$.

$$
\begin{aligned}
\int_{E} \psi d A & =\int_{E_{1} \backslash E_{2}} \psi d A+\int_{E_{2} \backslash E_{1}} \psi d A+\int_{E_{1} \cap E_{2}} \psi d A \\
& =\int_{E_{1} \backslash E_{2}} \psi_{1} d A+\int_{E_{2} \backslash E_{1}} \psi_{2} d A+\int_{E_{1} \cap E_{2}} \psi_{1} d A+\int_{E_{1} \cap E_{2}} \psi_{2} d A \\
& =\int_{E_{1}} \psi_{1} d A+\int_{E_{2}} \psi_{2} d A .
\end{aligned}
$$


For the general case, let $F=\bigcup_{j=1}^{n-1} E_{j}$ and let $\psi_{F}=\sum_{j=1}^{n-1} \psi_{j}$. Then

$$
\int_{E} \psi d A=\int_{F} \psi_{F} d A+\int_{E_{n}} \psi_{n} d A
$$

and the lemma follows by induction.

We now prove Theorem 1 .

Proof. Choose $f \in A_{0}(X)$ such that $f$ is analytic on $X$ and suppose that $\|f\|_{*} \leq 1$. For each $n$ let $K_{n}$ be a compact subset of $A_{n} \backslash X$ such that $f$ is analytic on $A_{n} \backslash K_{n}$. Since $f$ has a finite number of poles, we only need a finite number of $K_{n}$. Fix $n$ and let $\left\{Q_{j}\right\}$ be a covering of $K_{n}$ by dyadic squares so that no squares overlap except at their boundaries. Let $r_{j}$ denote the side length of $Q_{j}$. Let $Q_{j}^{*}=\frac{3}{2} Q_{j}$, the square with side length $\frac{3}{2} r_{j}$ and the same center as $Q_{j}$, and let $D_{n}=\bigcup Q_{j}^{*}$. Then by the Cauchy integral formula

$$
f^{(t)}\left(x_{0}\right)=\frac{t !}{2 \pi i} \sum_{n} \int_{\partial D_{n}} \frac{f(z)}{\left(z-x_{0}\right)^{t+1}} d z
$$

For each individual square $Q_{j}$, we can construct a smooth function $\phi_{j}$ such that $\phi_{j}$ has support on $Q_{j}^{*},\left\|\nabla \phi_{j}\right\|_{\infty} \leq C r_{j}^{-1}$, and $\sum_{j} \phi_{j}=1$ on a neighborhood of $\bigcup \phi_{j}$. Such a construction can be found in [6, Lemma 3.1]. Let $\phi=1-\sum_{j} \phi_{j}$. It then follows from Green's theorem and Lemma 3 that

$$
\begin{aligned}
\left|\frac{t !}{2 \pi i} \int_{\partial D_{n}} \frac{f(z)}{\left(z-x_{0}\right)^{t+1}} d z\right| & =\left|\frac{t !}{2 \pi i} \int_{\partial D_{n}} \frac{f(z) \phi(z)}{\left(z-x_{0}\right)^{t+1}} d z\right| \\
& =\left|\frac{t !}{\pi} \int_{D_{n}} \frac{f(z)}{\left(z-x_{0}\right)^{t+1}} \frac{\partial \phi}{\partial \bar{z}} d A\right| \\
& \leq \frac{t !}{\pi} \sum_{j}\left|\int_{Q_{j}^{*}} \frac{f(z)}{\left(z-x_{0}\right)^{t+1}} \frac{\partial \phi_{j}}{\partial \bar{z}} d A\right|
\end{aligned}
$$


Since $\int_{Q_{j}^{*}}\left(z-x_{0}\right)^{-(t+1)} \frac{\partial \phi_{j}}{\partial \bar{z}} d A=\int_{\partial Q_{j}^{*}}\left(z-x_{0}\right)^{-(t+1)} \phi_{j}(z) d z=0$, it follows that

$$
\begin{aligned}
\frac{t !}{\pi} \sum_{j}\left|\int_{Q_{j}^{*}} \frac{f(z)}{\left(z-x_{0}\right)^{t+1}} \frac{\partial \phi_{j}}{\partial \bar{z}} d A\right| & \leq C \sum_{j} \int_{Q_{j}^{*}}\left|f(z)-f_{Q_{j}^{*}}\right| \cdot\left|z-x_{0}\right|^{-(t+1)}\left|\frac{\partial \phi}{\partial \bar{z}}\right| d A \\
& \leq C 2^{(t+1) n} \sum_{j} m\left(Q_{j}^{*}\right) \Omega\left(f, Q_{j}^{*}\right) r_{j}^{-1} \\
& \leq C 2^{(t+1) n} \sum_{j} r_{j} \Omega_{f}\left(\frac{3}{2} r_{j}\right) .
\end{aligned}
$$

Since, $f \in V M O(\mathbb{C})$, the measure function $h(t)=t \Omega_{f}\left(\frac{3}{2} t\right)$ satisfies the conditions in the definition of $M_{*}^{1}$. Hence by taking the infimum over all such covers $\left\{Q_{j}\right\}$, we have that

$$
\left|\frac{t !}{2 \pi i} \int_{\partial D_{n}} \frac{f(z)}{\left(z-x_{0}\right)^{t+1}} d z\right| \leq C 2^{(t+1) n} M_{*}^{1}\left(K_{n}\right)
$$

and since $M_{*}^{1}$ is subadditive, it follows that

$$
\begin{aligned}
\left|f^{(t)}\left(x_{0}\right)\right| & \leq C \sum_{n=1}^{\infty} 2^{(t+1) n} M_{*}^{1}\left(K_{n}\right) \\
& \leq C \sum_{n=1}^{\infty} 2^{(t+1) n} M_{*}^{1}\left(A_{n} \backslash X\right) \\
& \leq C .
\end{aligned}
$$

If $g(z) \in V M O(\mathbb{C})$ is analytic on $X \cup\left\{x_{0}\right\}$, let $f(z)=\frac{g(z)}{\|g\|_{*}}$. Then $\|f\|_{*} \leq 1$ and hence $\left|f^{(t)}\left(x_{0}\right)\right| \leq C$. Thus $\left|g^{(t)}\left(x_{0}\right)\right| \leq C\|g\|_{*}$ and $A_{0}(X)$ admits a bounded point derivation of order $t$ at $x_{0}$.

To prove the converse, we can assume that $x_{0}=0$ and $X$ is entirely contained in the unit disk, and we suppose that

$$
\sum_{n=1}^{\infty} 2^{(t+1) n} M_{*}^{1}\left(A_{n} \backslash X\right)=\infty
$$


and we choose a decreasing sequence $\epsilon_{n}$ such that

$$
\sum_{n=1}^{\infty} 2^{(t+1) n} \epsilon_{n} M_{*}^{1}\left(A_{n} \backslash X\right)=\infty
$$

and $\left|2^{(t+1) n} \epsilon_{n} M_{*}^{1}\left(A_{n} \backslash X\right)\right| \leq 1$ for all $n$.

We now modify a construction used by Lord and O'Farrell for approximation in Lipschitz norms [10, pg.12]. It follows from Frostman's Lemma that for each $n$ there exists a positive measure $\nu_{n}$ with support on $A_{n} \backslash X$ such that

1. $\nu_{n}(B(z, r)) \leq \epsilon_{n} r$ for all balls $B$,

2. $\int \nu_{n}=C \epsilon_{n} M_{*}^{1}\left(A_{n} \backslash X\right)$.

Let

$$
f_{n}(z)=\int\left(\frac{\zeta}{|\zeta|}\right)^{t+1} \frac{d \nu_{n}(\zeta)}{\zeta-z}
$$

The same argument used in the proof of (b) of [9] (See also [13, pg.288].) shows that $f_{n}$ is analytic off $A_{n},\left\|f_{n}\right\|_{*} \leq C \epsilon_{n}$ and $f_{n} \in A_{0}(X)$. In addition,

$$
f_{n}^{(k)}(0)=t ! \int \frac{d \nu_{n}(\zeta)}{|\zeta|^{t+1}}
$$

Hence $f_{n}^{(t)}(0) \geq C 2^{(t+1) n} \epsilon_{n} M_{*}^{1}\left(A_{n} \backslash X\right)$. For each $n$ choose $p>n$ such that

$$
1 \leq \sum_{m=n}^{p} 2^{(t+1) m} \epsilon_{m} M_{*}^{1}\left(A_{m} \backslash X\right) \leq 2
$$

and let

$$
g_{n}(z)=\sum_{m=n}^{p} f_{m}(z)
$$


It follows that $g_{n}^{(t)}(0)$ is bounded below by a nonzero constant for all $n$. We wish to show that $\left\|g_{n}\right\|_{B M O(X)} \rightarrow 0$ as $n \rightarrow \infty$.

Let $Q$ be a cube in the annulus $A_{k}=\left\{2^{-(k+1)} \leq|z| \leq 2^{-k}\right\}$ and choose $f_{m}$ with $n \leq m \leq p$. Let $f_{m, Q}=\frac{1}{|Q|} \int_{Q} f_{m} d A$. Then there are 3 cases.

1. $k=m-1, m$, or $m+1$.

2. $k \geq m+2$

3. $k \leq m-2$.

If $k=m-1, m$, or $m+1$ then

$$
\Omega\left(f_{m}, Q\right)=\frac{1}{|Q|} \int_{Q}\left|f_{m}(z)-f_{m, Q}\right| d A_{z} \leq\left\|f_{m}\right\|_{*} \leq C \epsilon_{n}
$$

If $k \geq m+2$ then

$$
\begin{aligned}
\frac{1}{|Q|} \int_{Q}\left|f_{m}(z)-f_{m, Q}\right| d A_{z} & \leq 2\left\|f_{m}\right\|_{L^{\infty}\left(A_{k}\right)} \\
& \leq 2 \cdot 2^{m+2} \int \nu_{m} \\
& \leq C 2^{m} \epsilon_{m} M_{*}^{1}\left(A_{m} \backslash X\right) .
\end{aligned}
$$

If $k \leq m-2$ then

$$
\begin{aligned}
\frac{1}{|Q|} \int_{Q}\left|f_{m}(z)-f_{m, Q}\right| d A_{z} & \leq 2|| f_{m} \|_{L^{\infty}\left(A_{k}\right)} \\
& \leq 2 \cdot 2^{m-2} \int \nu_{m} \\
& \leq C 2^{m} \epsilon_{m} M_{*}^{1}\left(A_{m} \backslash X\right) .
\end{aligned}
$$

It follows from the triangle inequality that 


$$
\begin{aligned}
\left\|g_{n}\right\|_{*} & \leq \sum_{m=n}^{p}\left\|f_{m}\right\|_{*} \\
& \leq 3 C \epsilon_{n}+C \sum_{m=n}^{p} 2^{m} \epsilon_{m} M_{*}^{1}\left(A_{m} \backslash X\right) \\
& \leq 3 C \epsilon_{n}+C 2^{-n} \sum_{m=n}^{p} 4^{m} \epsilon_{m} M_{*}^{1}\left(A_{m} \backslash X\right) \\
& \leq C\left(3 \epsilon_{n}+2^{-n}\right) .
\end{aligned}
$$

Moreover from Fubini's theorem,

$$
\begin{aligned}
\left\|f_{m}\right\|_{L^{1}(X)} & =\int_{X}\left|\int\left(\frac{\zeta}{|\zeta|}\right)^{t+1} \frac{d \nu_{m}(\zeta)}{\zeta-z}\right| d A(z) \\
& \leq \int_{X} \int \frac{d \nu_{m}(\zeta)}{|\zeta-z|} d A \\
& =\iint_{X} \frac{1}{|\zeta-z|} d A(z) d \nu_{m}(\zeta)
\end{aligned}
$$

Fix $\zeta$ and let $B_{r}$ denote the ball with radius $r$ centered at $\zeta$, so that the area of $B_{r}$ is the same as the area of $X$. Then

$$
\begin{aligned}
\iint_{X} \frac{1}{|\zeta-z|} d A(z) d \nu_{m}(\zeta) & \leq \iint_{B_{r}} \frac{1}{|\zeta-z|} d A(z) d \nu_{m}(\zeta) \\
& \leq 2 \pi r \int d \nu_{m}(\zeta) \\
& \leq C \epsilon_{m} M_{*}^{1}\left(A_{m} \backslash X\right)
\end{aligned}
$$

Thus, $\left\|g_{n}\right\|_{L^{1}(X)} \leq C \sum_{m=n}^{p} \epsilon_{m} M_{*}^{1}\left(A_{m} \backslash X\right) \leq C$. Hence $\left\|g_{n}\right\|_{B M O(X)} \rightarrow 0$ as $n \rightarrow \infty$, but $g_{n}^{(t)}(0)$ is bounded away from 0 for all $n$. Hence $A_{0}(X)$ does not admit a bounded point derivation at 0. 


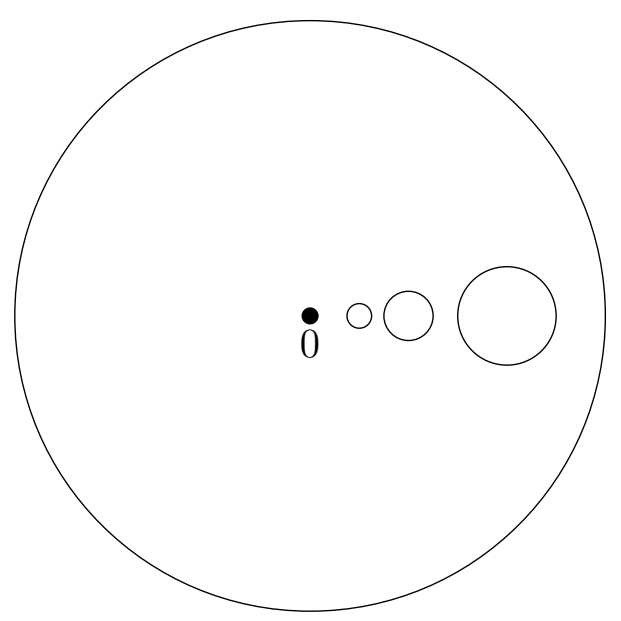

Figure 1: A roadrunner set

\section{$5 \quad$ Examples}

In this section, we will construct some examples for which Theorem 1 applies. The first construction is that of a set $X$ such that $A_{0}(X)$ does not admit a bounded point derivation at 0 but such that $A_{\alpha}(X)$ admits a bounded point derivation at 0 for all $\alpha>0$.

Let $D$ be the open unit disk and let $A_{n}=\left\{2^{-(n+1)} \leq|z| \leq 2^{-n}\right\}$. For each $n$, let $B_{n}$ be a closed disk contained entirely in $A_{n}$ with radius $r_{n}=4^{-n} n^{-1}$ and let $X=D \backslash \bigcup B_{n}$. This is known as a roadrunner set. See figure 1 .

Since $M_{*}^{1}\left(A_{n} \backslash X\right)=M_{*}^{1}\left(B_{n}\right)=r_{n}$, it follows that

$$
\sum_{n=1}^{\infty} 4^{n} M_{*}^{1}\left(A_{n} \backslash X\right)=\sum_{n=1}^{\infty} n^{-1}=\infty
$$

and thus $A_{0}(X)$ does not admit a bounded point derivation at 0 . However, $M_{*}^{1+\alpha}\left(A_{n} \backslash X\right)=$ $M_{*}^{1+\alpha}\left(B_{n}\right)=r_{n}^{1+\alpha}$. Hence

$$
\sum_{n=1}^{\infty} 4^{n} M_{*}^{1+\alpha}\left(A_{n} \backslash X\right)=\sum_{n=1}^{\infty} 4^{-n \alpha} n^{-(1+\alpha)}<\infty
$$

and thus $A_{\alpha}(X)$ admits a bounded point derivation at 0 for all $\alpha>0$.

For an example of a set for which $A_{0}(X)$ admits a bounded point derivation at 0 , we can modify the previous construction so that the removed disks $B_{n}$ have radii $r_{n}=4^{-n} n^{-2}$. Then 


$$
\sum_{n=1}^{\infty} 4^{n} M_{*}^{1}\left(A_{n} \backslash X\right)=\sum_{n=1}^{\infty} n^{-2}<\infty
$$

\section{References}

[1] Boivin, B. and Verdera, J. Approximation par fonctions holomorphes dans les espaces $L^{p}$, Lipa et BMO. (French) Indiana Univ. Math. J. Vol. 40, No. 2 (1991) 393-418

[2] Bonilla, A. and Fariña, J.C. Meromorphic and entire approximation in BMO-norm J. Approx. Theory 76, (1994) 203-218

[3] Fernström, C. and Polking, J.C. Bounded point evaluations and approximation in $L^{p}$ by solutions of elliptic partial differential equations. J. Functional Analysis 28 (1978), no. 1, 1-20

[4] Garnett, J.B. Analytic Capacity and Measure Lecture Notes in Math., vol. 1043, SpringerVerlag, Berlin and New York, 1972.

[5] Hallstrom, A.P., On bounded point derivations and analytic capacity. J. Functional Analysis 3 (1969) 35-47

[6] Harvey, R. and Polking, J. Removable singularities of solutions of linear partial differential equations Acta Math. 125 (1970), 39-56

[7] Hedberg, L.I. Bounded point evaluations and capacity. J. Functional Analysis 10 (1972), 269-280

[8] John, F. and Nirenberg, L. On functions of bounded mean oscillation. Comm. Pure Appl. Math. 14 (1961), 415-426

[9] Kaufman, R. Hausdorff measure, BMO, and analytic functions Pacific J. Math. 102 (1982) 369-371

[10] Lord, D.J. and O'Farrell, A.G. Boundary smoothness properties of Lipa analytic functions. J. Anal. Math. 63 (1994), 103119.

[11] Meyers, N.G. Mean oscillation over cubes and Hölder continuity. Proc. Amer. Math. Soc, Vol. 15 No. 5 (1964), 717-721 
[12] O'Farrell, A.G. Boundary values of holomorphic distributions in negative Lipschitz classes. Preprint 2019. arXiv 1806.09979

[13] Verdera, J. BMO rational approximation and one-dimensional Hausdorff content Trans. Amer. Math. Soc. Vol. 297 No. 1 (1986), 283-304. 